\title{
STAT3 regulates hypoxia-induced epithelial mesenchymal transition in oesophageal squamous cell cancer
}

\author{
YAO CUI ${ }^{1,2}$, YUN-YUN LI $^{3}$, JIAN LI $^{4}$, HONG-YAN ZHANG ${ }^{1}$, FENG WANG $^{1}$, XUE BAI $^{1}$ and SHAN-SHAN LI ${ }^{1}$ \\ ${ }^{1}$ Department of Pathology, The First Affiliated Hospital of Zhengzhou University and Basic Medical College of \\ Zhengzhou University, Zhengzhou, Henan 450052; ${ }^{2}$ Department of Oncology, Henan Provincial People's Hospital \\ (Zhengzhou University People's Hospital), Zhengzhou, Henan 450003; ${ }^{3}$ Department of Stomatology, The First Affiliated \\ Hospital of Zhengzhou University, Zhengzhou, Henan 450052; ${ }^{4}$ Department of General Surgery, \\ Henan Cancer Hospital, Zhengzhou University, Zhengzhou, Henan 450003, P.R. China
}

Received January 24, 2016; Accepted March 29, 2016

DOI: $10.3892 /$ or.2016.4822

\begin{abstract}
Hypoxia plays a key role in tumour initiation and metastasis; one of the mechanisms is to induce epithelialmesenchymal transition (EMT). Signal transducer and activator of transcription 3 (STAT3) is involved in EMT by regulating the transcriptional regulators of E-cadherin, the biomarker of EMT. Until now, however, few studies have focused on the effects of STAT3 in hypoxia-induced EMT in tumour cells. The goal of this study was to investigate the roles of STAT3 in hypoxia-induced EMT in oesophageal squamous cell carcinoma (ESCC). The ESCC cells, TE-1 and EC-1, were incubated in normoxia, or in $\mathrm{CoCl}_{2}$, which was used to mimic hypoxia. With $\mathrm{CoCl}_{2}$, the ESCC cells showed increased migration and invasion abilities, accompanied with upregulation of HIF-1 $\alpha$, STAT3, and vimentin, and downregulation of E-cadherin. Knockdown of STAT3 inhibited EMT of ESCC cells and downregulated HIF-1 $\alpha$ in vitro and in vivo. In ChIP assays, STAT3 bound to the promoter of HIF-1 $\alpha$, suggesting that STAT3 regulates transcription of $H I F-1 \alpha$. In conclusion, hypoxia induces EMT of ESCC, and STAT3 regulates this process by promoting $H I F-1 \alpha$ expression.
\end{abstract}

\section{Introduction}

Hypoxic microenvironments exist in various malignant carcinomas, leading to angiogenesis, tumourigenesis, and metastasis of neoplasms. One of the mechanisms is that hypoxia can induce epithelial-mesenchymal transition (EMT), a crucial step in embryological development and cancer progression.

Correspondence to: Professor Shan-Shan Li, Department of Pathology, The First Affiliated Hospital of Zhengzhou University and Basic Medical College of Zhengzhou University, 1 Jianshe East Road, Zhengzhou, Henan 450052, P.R. China

E-mail: 1sspath@163.com

Key words: STAT3, epithelial-mesenchymal transition, hypoxia, oesophageal squamous cell carcinoma
In EMT, the epithelial cells lose cell-to-cell tight junctions and baso-apical polarity, adopt mesenchymal cell phenotypes and plasticity, and become mobile $(1,2)$. Loss of the epithelial markers, E-cadherin and $\beta$-catenin, and acquisition of the mesenchymal markers, vimentin, $\mathrm{N}$-cadherin, and fibronectin, are the molecular hallmarks of EMT.

Hypoxia-induced EMT was found in a number of carcinomas, including colon, breast, pancreatic, and ovarian carcinomas, and hepatoblastoma (3). Hypoxia inducible factors (HIFs) regulate hypoxic responses, including malignant biological behaviour in tumour cells. HIF-1 is the most important factor, and is composed of two subunits, HIF-1 $\alpha$ and HIF-1 $\beta$. The HIF-1 $\alpha$ subunit is regulated by oxygen tension, whereas HIF-1 $\beta$ is constitutively expressed. HIF-1 targets the hypoxia-responsive genes (HRE), including the transcriptional regulators (Snail, Slug, Twist, ZEB) of E-cadherin (4). It has been proposed that long-term hypoxia drives a perpetual EMT through upregulation of the zinc finger, E-box binding, homeobox protein, ZEB2, whereas short-term hypoxia induces a reversible EMT that requires Twist1 (5). Hypoxia also is involved in EMT-triggering pathways of TGF- $\beta$, Notch, NFאB, Wnt, and PI3K (6-10). However, the underlying mechanisms of how these multigene interactive cascades are triggered are still unknown.

Signal transducer and activator of transcription 3 (STAT3), a member of the STAT family of transcription factors that is activated through various mitogenic signalling pathways of cell growth, migration, and angiogenesis, is overexpressed and constitutively activated in a variety of human malignancies (11). Studies have demonstrated that STAT3 is required for EMT. TGF- $\beta$ induced EMT in pancreatic cancer requires STAT3 activity and Smad4-dependent signalling regulates this process (12). Activation of EGFR or IL-6R results in high levels of STAT3 and activation of the downstream JAK2/STAT3 pathway, inducing a fibroblast-like morphology and migratory phenotype, consistent with the upregulation of mesenchyme-associated $\mathrm{N}$-cadherin and vimentin, and the nuclear translocation of $\beta$-catenin (13). EGF promotes EMT of cancer cells through a ROS/STAT3/HIF-1 $\alpha /$ TWIST1/ $\mathrm{N}$-cadherin signalling cascade (14). STAT3 directly regulates the expression of genes encoding the transcription factors 
TWIST, ZEB1, and Snail (15-17). STAT3 and associated EMT may contribute to chemoresistance, by involving EGFR signalling $(18,19)$.

Overexpression of HIF- $1 \alpha$ and STAT3 has been detected in numerous cancers, and these proteins play important roles in cancer progression and migration. Activated STAT3 increases HIF-1 $\alpha$ protein levels by enhancing HIF-1 $\alpha$ stability and accelerating its de novo synthesis (20). STAT3 activates HIF1 target genes by binding to their promoters, interacting with HIF1 $\alpha$, and recruiting the coactivators, CREB binding protein (CBP) and p300, and RNA polymerase II (Pol II) to form enhanceosome complexes (21). Stat3 regulates expression of Akt, which is required for growth signal-induced HIF-1 upregulation (22). However, the role of STAT3 in hypoxia-induced EMT has not been reported.

Oesophageal carcinoma is the fourth most prevalent malignancy in China and the sixth cause of cancer-related death in the world. Oesophageal squamous cell carcinoma (ESCC) is one of the major histopathological subtypes of oesophageal cancer. Metastasis and chemo/radio resistance of ESCC are closely related to hypoxic conditions and EMT. This study investigated the mechanisms of hypoxia-induced EMT in ESCC and the roles of STAT3 in this process.

\section{Materials and methods}

Cell culture. Human ESCC cell lines TE-1 and EC-1 were purchased from the Shanghai Institute of Biochemistry and Cell Biology (Shanghai, China). The cells were grown in RPMI-1640 supplemented with $10 \%$ foetal bovine serum (FBS) and 1\% penicillin/streptomycin. The cells were incubated under the normoxic condition of $20 \% \mathrm{O}_{2}$, or in the presence of cobalt chloride $\left(\mathrm{CoCl}_{2}\right.$; Sigma, St. Louis, USA), a known hypoxia-inducing agent (23).

Reverse transcription-PCR (RT-PCR) and real-time PCR $(q P C R)$. Total RNA Extraction, RT-PCR, and qPCR were performed as previously described (3). Total cellular RNA was extracted with the Ultrapure RNA kit (CW Biotech Co. Beijing, China). First-strand cDNA was synthesized with oligo (dT) primers (Sangon Biotech. Shanghai, China) and the Super RT cDNA kit (CW Biotech Co.). The primer sequences were as follows: E-cadherin forward: 5'-GAGAACGCATTGCC ACATACAC-3', reverse: 5'-GAGCACCTTCCATGACAGA CCC-3'; vimentin forward: 5'-ATGTGGATGTTTCCAAG CCTGAC-3', reverse: 5'-GAGTGGGTATCAACCAGAGG GAGT-3'; HIF-1 $\alpha$ forward: 5'-CACTGCACAGGCCACA TTCACGT-3', reverse: 5'-GAGCACCTTCCATGACAGA CCC-3'; STAT3 forward: 5'-GAGAACGCATTGCCACAT ACAC-3', reverse: 5'-TCTGGCCGACAATACTTTCC-3'; and $\beta$-actin forward: 5'-GTCCACCGCAAATGCTTCTA-3', reverse: 5'-TGCTGTCACCTTCACCGTTC-3'. Products from RT-PCR were monitored by polyacrylamide gel electrophoresis (PAGE). qPCR was performed using the UltraSYBR Mixture (CW Biotech Co.) and ABI 7300 Real-time PCR system. To normalize the amount of input RNA, PCR was performed with probe and primers for $\beta$-actin.

Immunoblotting. Cells were harvested and incubated with RIPA buffer for $10 \mathrm{~min}$ to collect the cell lysates. The total cellular protein was separated using 10\% SDS-PAGE and transferred to PVDF membranes (Millipore Corp., Bedford, MA, USA). PVDF membranes were blocked with TRIS buffered saline (TBS) containing 5\% non-fat milk, and then incubated with the primary antibody at $4^{\circ} \mathrm{C}$ overnight, followed by incubation with goat anti-rabbit/mouse secondary antibodies (1:10000, Odessey, China). The primary antibodies included anti-HIF-1 $\alpha$ (1:1000, Abcam), anti-E-cadherin (1:2000, Abcam), anti- $\beta$-actin (1:10000, Sigma), anti-STAT3 (1:2000, Santa Cruz Biotechnology, Santa Cruz, CA, USA), anti-p-STAT3 (Tyr705) (1:2000, Santa Cruz Biotechnology), and anti-vimentin (1:2000, Abcam), anti-GAPDH (1:1000, Santa Cruz Biotechnology). Protein-antibody complexes were visualised using the ECL detection system (Mbchem, Shanghai, China) following the manufacturer's protocol. $\beta$-actin or GAPDH was used as an internal control.

Immunofluorescence. The cells were grown on coverslips in 24-well plates. Before staining, the cells were fixed with $4 \%$ formaldehyde for $10 \mathrm{~min}$, followed by $0.2-0.3 \%$ Triton X-100 for $10 \mathrm{~min}$ and then were blocked in $1 \%$ BSA for $1 \mathrm{~h}$. For staining, the cells were incubated with the primary antibodies monoclonal anti-E-cadherin (1:200, Abcam), anti-vimentin (1:200, Abcam) or anti-HIF-1 $\alpha$ (1:200, Abcam) for $1 \mathrm{~h}$ and then with goat anti-rabbit/mouse secondary antibodies (1:1000, Invitrogen, Carlsbad, CA, USA) for $1 \mathrm{~h}$ at room temperature. The nuclei of the cells were counterstained with DAPI (1:10000, Invitrogen). After each step, cells were washed with PBS. The images were acquired by laser scanning microscopy (Zeiss, Jena, Germany).

Immunohistochemistry. E-cadherin, vimentin, HIF-1 $\alpha$, or pSTAT3 protein expression in tissue was detected by immunohistochemistry. Sections $(4 \mu \mathrm{m})$ were $10 \%$ formalinfixed, paraffin-embedded, deparaffinized in xylene, and then rehydrated through graded ethanol solutions. The slides were incubated in $10 \mathrm{mM}$ citrate buffer $\left(\mathrm{pH} \mathrm{6.0)}\right.$ at $95^{\circ} \mathrm{C}$ for $20 \mathrm{~min}$, followed by $3 \%$ hydrogen peroxide in methanol for $10 \mathrm{~min}$, and then were blocked with $10 \%$ bull serum albumin for $10 \mathrm{~min}$ in room temperature. The tissue slides were incubated with anti-E-cadherin (1:100, Santa Cruz Biotechnology), anti-vimentin (1:100, Santa Cruz Biotechnology), anti-HIF-1 $\alpha$ (1:100, Santa Cruz Biotechnology), or anti-pSTAT3 antibody (1:100, Santa Cruz Biotechnology) at $4^{\circ} \mathrm{C}$ overnight, and with goat anti-rabbit/mouse secondary antibody (1:200, Santa Cruz Biotechnology) for $30 \mathrm{~min}$ at room temperature. The sites of antibody binding were visualized by $0.05 \%$ diaminobenzidine (DAB) and stained with Mayer's hematoxylin. The slides were scored in a blinded manner by two independent pathologists. The intensity of protein expression was calculated by summing the percentage of positively stained cells and the staining intensity.

Small interfering RNA (siRNA) and Small hairpin RNA (shRNA) transfection. Human HIF-1 $\alpha$ siRNA (Santa Cruz Biotechnology) and STAT3 shRNA-expressing plasmid vector were used in the study. The oligonucleotide encoding an shRNA targeting 5'-TGTTCTCTATCAGCACAAT-3', starting at position 466 in the STAT3 sequence (GenBank Accession number: NM 39276) was cloned between the HpaI 
and XhoI restriction sites of the plasmid pGCL-GFP, which containing a CMV-driven GFP reporter (24), to constructed a vector mediating RNAi (RNA interference) against STAT3 (pGCL-GFP-STAT3siRNA). HIF-1 $\alpha$ siRNA, empty vector PGCL-GFP, or pGCL-GFP-STAT3siRNA was transfected into cells using Lipofectamine 2000 (Invitrogen) following the manufacturer's instructions.

Chromatin immunoprecipitation (ChIP). TE-1 and EC-1 cells were cultured in $100-\mathrm{mm}$ dishes at a density of $1 \times 10^{6} / \mathrm{dish}$, serum-starved overnight, and then cross-linked with $1 \%$ formaldehyde. Chromatin preparation and immunoprecipitation were as previously described $(15,25)$. Chromatin was fragmented by sonication and was incubated with anti-STAT3 (Santa Cruz Biotechnology) for the experiment, anti-RNA polymerase II (Pol II) (Santa Cruz Biotechnology) for the positive control, or rabbit polyclonal $\operatorname{IgG}$ (Santa Cruz Biotechnology) for the negative control for $3 \mathrm{~h}$ at $4^{\circ} \mathrm{C}$. Immunoprecipitation complex was collected by vortexing with protein $\mathrm{A} / \mathrm{G}$ agarose and extensive washed, eluted, and purified. Then the DNA was used directly as template for PCR detection. The possible binding sites of HIF-1 $\alpha$ were analysed using Primer Premier 5.0, DNA star and BLAST. Eight pairs of primers for fragments covering the $3 \mathrm{kbp}$ of the promoter of the HIF-1 $\alpha$ gene were designed: region 1 forward: 5'-GATATACTTA CATAGTAATAA-3', reverse: 5'-TGGATCATTTCAAAAG TTTAA-3'; region 2 forward: 5'-GACTTAGATTTACTGT TACAA-3', reverse: 5'-TTTTAGTAGAGACGAGGTTTC-3'; region 3 forward: 5'-AAAATTATCCGAGTGTGGTG-3', reverse: 5'-CTTTTTAGTTTAACATTCTCA-3'; region 4 forward: 5'-GTATTCTTTCCACAATTAAAA-3', reverse: 5'-TAGTGGCTTTCAATTGTATTA-3'; region 5 forward: 5'-GATATTTATATTTACAACCCA-3', reverse: 5'-ATTACC TAACCATGGGTCAAT-3'; region 6 forward: 5'-TAGGTA ATCTGGTAAGGAAA-3', reverse: 5'-CTCTCAGCCAATC AGGAGGC-3'; region 7 forward: 5'-GGACTTGCCGCC TGCGTCGC-3', reverse: 5'-TCCCTCCACACGCGGAG AAGA-3'; region 8 forward: 5'-GAACCCGCCTCCACCTC AGGT-3', reverse: 5'-ATGTCTTCACGGCGGGCGGCC-3'.

Wound healing migration assay. TE-1 and EC-1 were seeded in 6 -well plates, and wounds were made by scratching the plates with sterile $200 \mu$ l pipet tips, five scratches per well. Cells were allowed to continue growing in normoxia or with $\mathrm{CoCl}_{2}(100 \mu \mathrm{mol} / \mathrm{l})$ in $5 \% \mathrm{CO}_{2}$ at $37^{\circ} \mathrm{C}$. Images were taken at different times. The widths of the scratches were measured at five different regions and are presented as mean \pm SD.

Invasion assay. Twenty-four-well invasion chambers (Corning Inc., Corning, NY, USA) coated with Matrigel (BD Biocoat) were used as described previously (25). Starved cells (cultured in serum-free medium for $12 \mathrm{~h}$ before use) were added to the rehydrated inserts with serum-free RPMI1640, and 10\% FBS RPMI-1640 was added into the wells. One well of each plate was left as a blank. The chambers were incubated in normoxia or with $\mathrm{CoCl}_{2}(100 \mu \mathrm{mol} / \mathrm{l})$ for $12 \mathrm{~h}$. The cells that passed through the filters to the lower surface of the insert chambers were stained with $0.1 \%$ crystal violet, and then counted.
Tumour xenografts. All animal work was done in accordance with a protocol approved by the Laboratory Animal Management Committee of Zhengzhou University. Six-week-old male, athymic, nude mice were used for the xenografting experiment. EC-1 $\left(1 \times 10^{6}\right)$ cells, mixed with an equal volume of Matrigel, were implanted subcutaneously into the dorsal regions of mice. Mice were treated with normal saline (as control), empty vector pGCL-GFP, or STAT3 siRNA by tail vein injection from day 7 after tumour implantation, twice a week. PGCL-GFP or STAT3 siRNA was mixed with lipofecter (Beyotime, Jiangsu, China) before injection $(26,27)$. Four weeks after implantation, mice were euthanized and the tumour sizes were measured. Expression of pSTAT3, HIF-1 $\alpha$, E-cadherin, and vimentin in the xenografts tumour was detected by immunohistochemistry.

Statistical analyses. Statistical analyses were performed using IBM SPSS Statistics 21.0 (SPSS Inc. IBM, Armonk, NY, USA). Differences between two groups were assessed using the Student's t-test. Differences among three or more groups were evaluated using one-way ANOVAs. Data are shown as mean \pm SD from three independent experiments. P-values $<0.05$ were considered statistically significant.

\section{Results}

Hypoxia induces EMT and activates STAT3 in ESCC cells. Hypoxia induces several kinds of tumour cells to transform from epithelium to mesenchyme, both morphologically and biologically. The tumours include pancreatic, breast, ovarian, colon and hepatocellular cancers $(28,29)$. However, there are few studies focusing on hypoxia-induced EMT in ESCC. To explore the effects of hypoxia on ESCC cells, we observed the morphological changes and the migration and invasion abilities of the ESCC cells, EC-1 and TE-1, in normoxia or hypoxia. The ESCC cells had the morphology of mesenchymal cells: spindle shaped with more pseudopodia in hypoxia or with $\mathrm{CoCl}_{2}$ (Fig. 1A). The migration and invasion rates of ESCC cells were significantly enhanced in the hypoxia mimic, $\mathrm{CoCl}_{2}$, than in normoxia $(\mathrm{P}<0.05$; Fig. 1B and C). The EMT biomarkers, E-cadherin and vimentin, and the hypoxic-response marker, HIF-1 $\alpha$, were detected in ESCC cells in normoxia and hypoxia. When exposed to $\mathrm{CoCl}_{2}$, mRNA and protein expression of E-cadherin decreased and vimentin increased to levels higher than those in normoxia. HIF-1 $\alpha$ protein, but not mRNA, increased more sharply with $\mathrm{CoCl}_{2}$ than in normoxia. Phosphorylated STAT3, the active state of STAT3, increased in hypoxia (Fig. 1D and E), which was consistent with earlier studies $(30,31)$. Changes in EMT protein and HIF- $1 \alpha$ expression in $\mathrm{CoCl}_{2}$ were confirmed by immunofluorescence assay (Fig. 1F). Previous studies have shown that protein levels of HIF-1 $\alpha$ and pSTAT3 increase with $\mathrm{CoCl}_{2}$ as they do in 'real' hypoxia, and that tumour cells performed similar EMTs in the hypoxia mimic, $\mathrm{CoCl}_{2}$. Thus, the above molecular changes were not only due to $\mathrm{CoCl}_{2}$ treatment, but also were reflective of changes in 'real' hypoxia.

STAT3 regulates HIF-1 $\alpha$ and EMT in hypoxia. STAT3 plays important roles in EMT induced by TGF $\beta$, EGF, or IL-6 $(32,33)$. To determine the effects of STAT3 on EMT induced by hypoxia, we used STAT3 siRNA to knockdown STAT3 in 
A

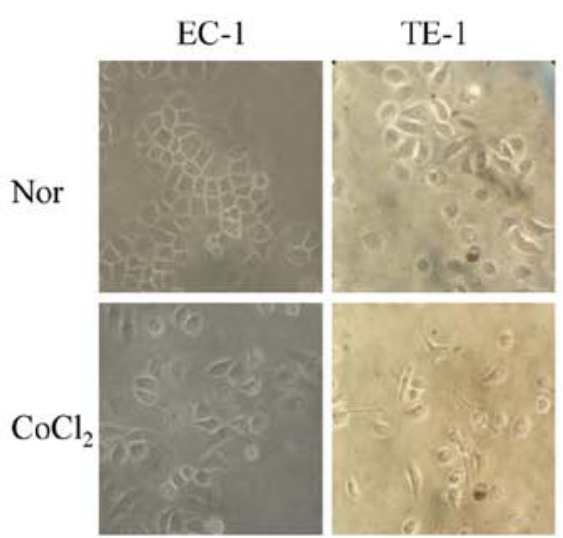

B
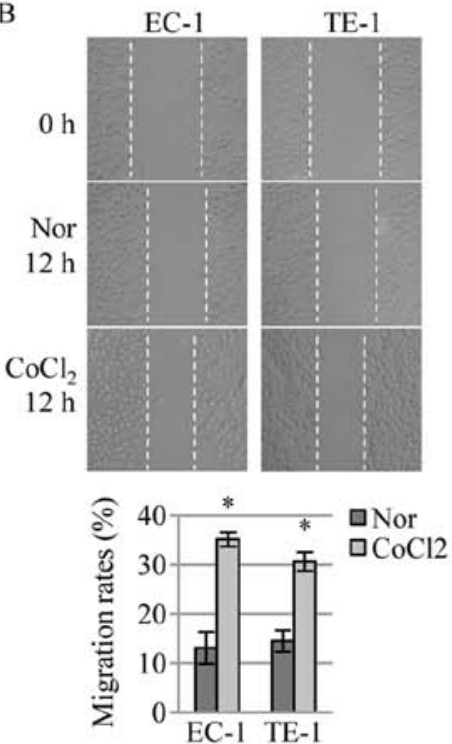

C

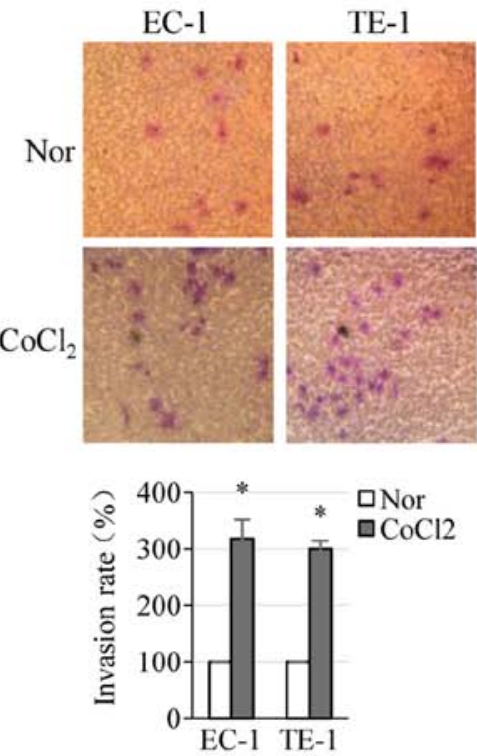

D

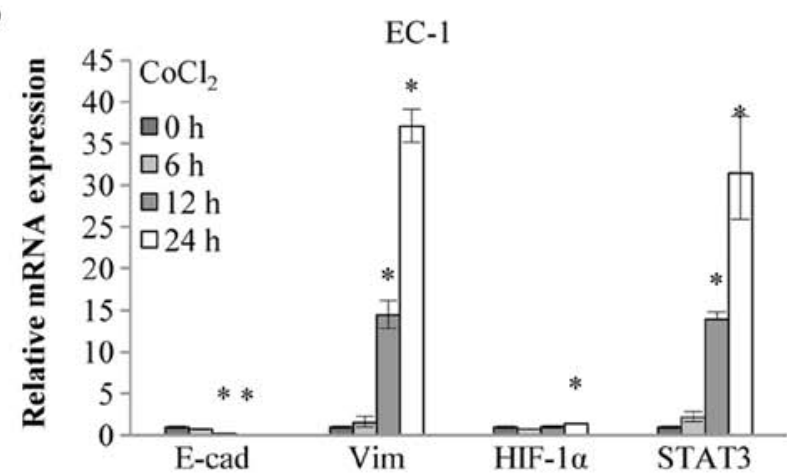

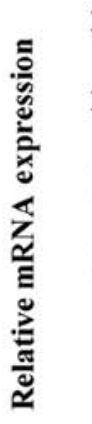

${ }^{25} 7 \mathrm{CoCl}_{2}$

$20-0 \mathrm{~h}$

$\square 6 \mathrm{~h}$

$5-\square 12 \mathrm{~h}$

TE-1

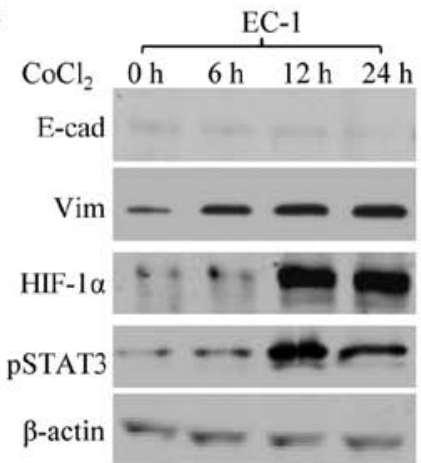

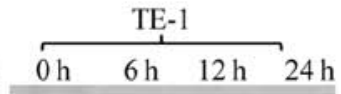

$\mathrm{F}$
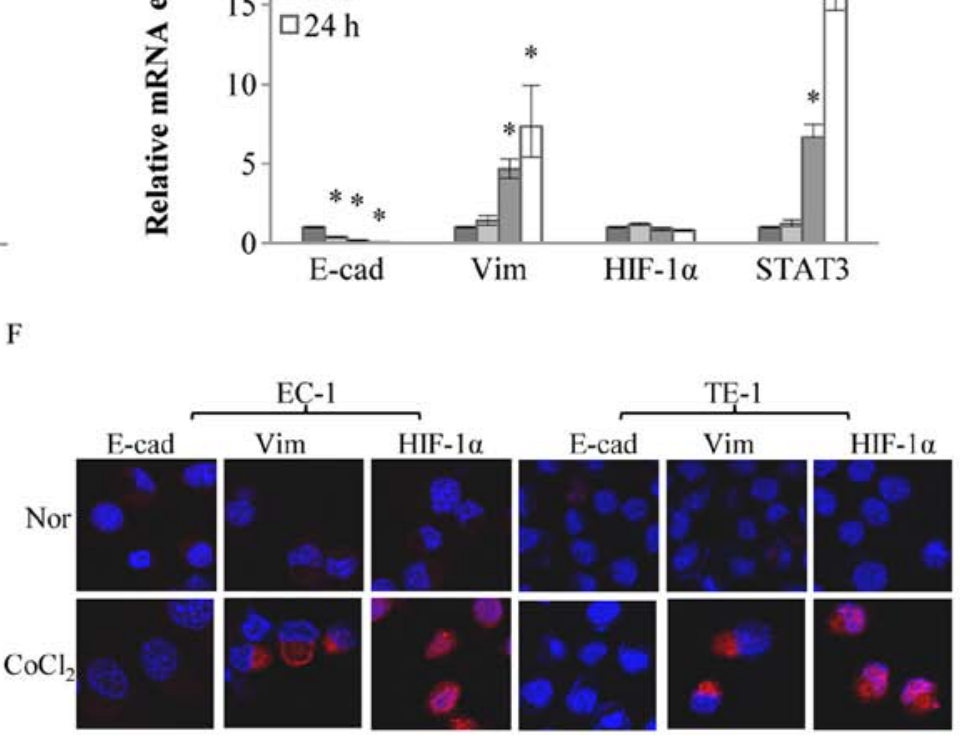

Figure 1. Hypoxia induces EMT and activates STAT3 in ESCC cells. Human ESCC EC-1 and TE-1 were used. (A) CoCl $\mathrm{C}_{2}$-treated ESCC cells displayed mesenchymal morphology. Cells were cultured in $100-\mathrm{mm}$ dishes at $6 \times 10^{6}$ per dish in normoxia (Nor) or with $\mathrm{CoCl}_{2}(100 \mu$ mol/1). EC-1 and TE-1 cells displayed mesenchymal-like morphology when treated with $\mathrm{CoCl}_{2} 24$ h. (B) Hypoxia accelerates migration of ESCC cells. EC-1 and TE-1 cells were incubated in the 6 -well plates and scratches were made. The cells were cultured in normoxia or with $\mathrm{CoCl}_{2}(100 \mu \mathrm{mol} / \mathrm{l})$ for $12 \mathrm{~h}$ and the migration rates were calculated. The cell migration rate in $\mathrm{CoCl}_{2}$ was significantly higher than in normal oxygen $\left({ }^{*} \mathrm{P}<0.05\right)$. (C) Hypoxia accelerates invasion by ESCC cells. Cells were seeded into Matrigel coated 24-well invasion chambers, incubated in normoxia or with $\mathrm{CoCl}_{2}(100 \mu \mathrm{mol} / \mathrm{l})$ for $12 \mathrm{~h}$. Penetrating cells were stained with $0.1 \%$ crystal violet. The cell invasion rate in $\mathrm{CoCl}_{2}$ was significantly higher than in normal oxygen ("P<0.05). (D) Relative mRNA expression levels of E-cadherin (E-cad), vimentin (vim), HIF-1 $\alpha$, and STAT3 in ESCC cells treated with or without $\mathrm{CoCl}_{2}(100 \mu \mathrm{mol} / 1)$. mRNA expression with $\mathrm{CoCl}_{2}$ was significantly different from that in normal oxygen $(" \mathrm{P}<0.05)$. (E) Effects of hypoxia on expression of E-cadherin, vimentin, HIF-1 $\alpha$, and pSTAT3. Western blot analysis of the protein levels in ESCC cells incubated with or without $\mathrm{CoCl}_{2}(100 \mu \mathrm{mol} / 1)$. Results were normalized against $\beta$-actin. (F) IF of E-cadherin, vimentin, and HIF-1 $\alpha$ expression of ESCC cells. Cells were grown on coverslips in 24-well plates with or without $\mathrm{CoCl}_{2}(100 \mu \mathrm{mol} / \mathrm{l})$ for $24 \mathrm{~h}$ and then were stained with antibodies. Nuclei (blue) were stained by DAPI. The results represent means \pm SD from three independent experiments.

ESCC EC-1 cells. STAT3 mRNA and phosphorylated protein were effectively silenced by STAT3 siRNA, both in normoxia and in hypoxia. STAT3 siRNA downregulated HIF-1 $\alpha$ mRNA and protein expression in normoxia and hypoxia. The EMT markers E-cadherin and vimentin were upregulated and downregulated $(\mathrm{P}<0.05)$, respectively, and the tendencies were more evident in hypoxia, when transfected with STAT3 siRNA (Fig. 2A-C). The invasion and migration rates of the cells declined when treated with STAT3 siRNA $(\mathrm{P}<0.05)$, and this effect was stronger in hypoxia (Fig. 2D and E). The above 
A

E-cadherin

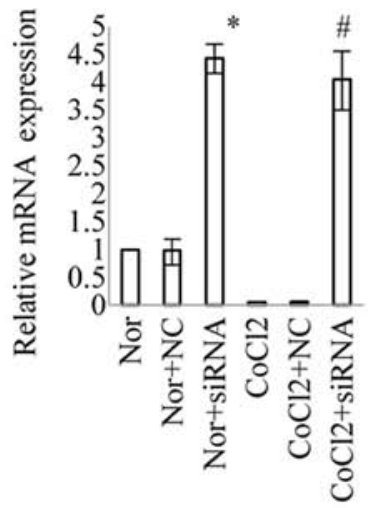

Vimentin

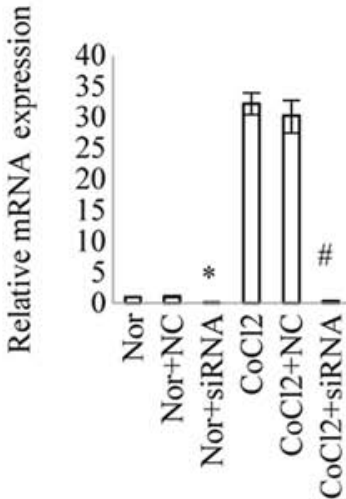

HIF- $1 \alpha$

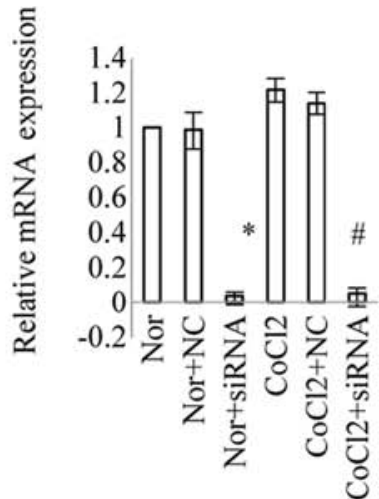

STAT3

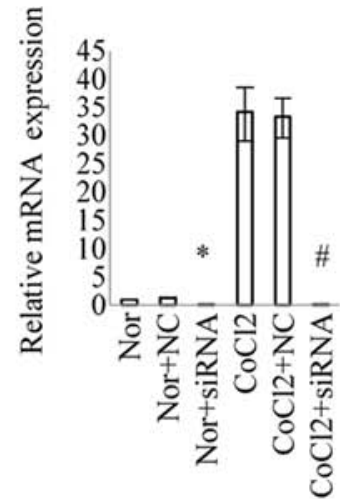

B

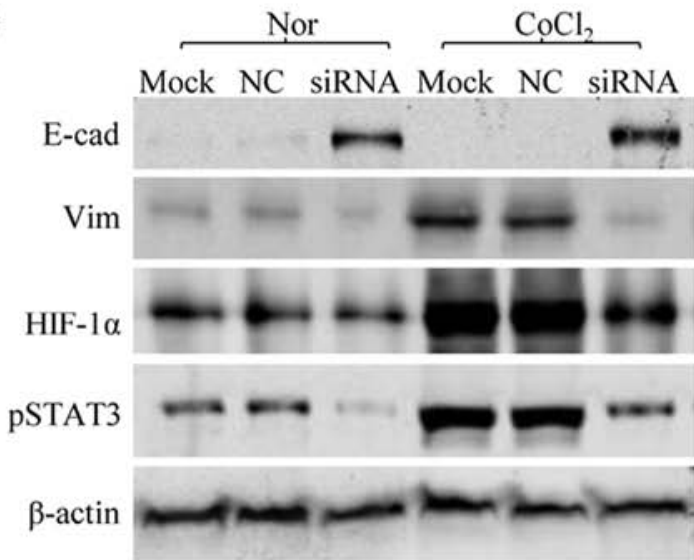

C

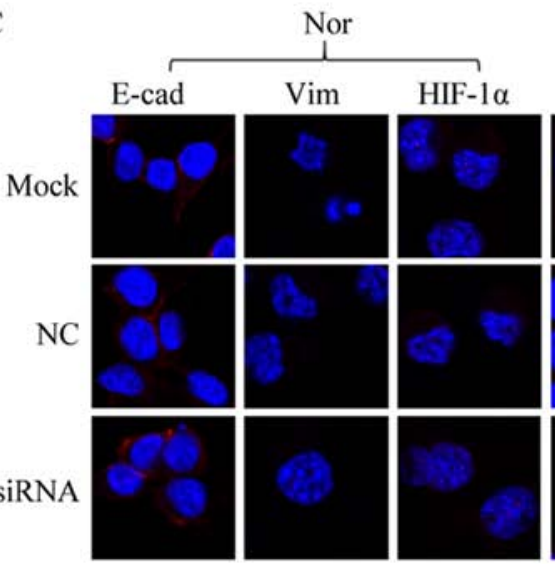

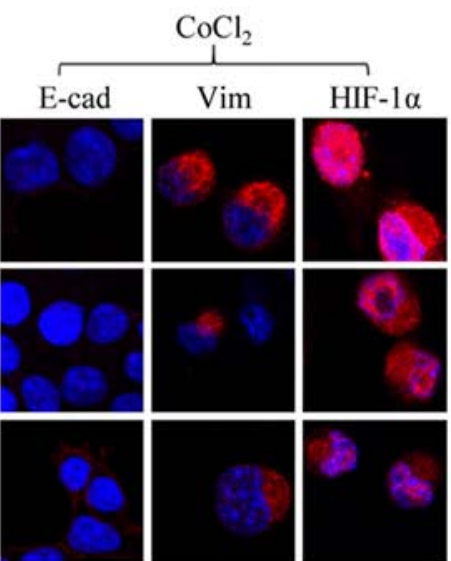

D
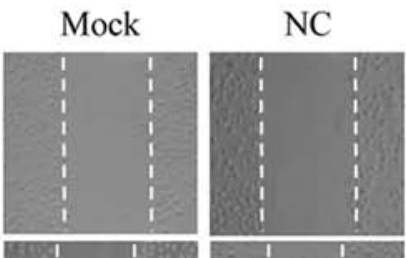
siRNA

$0 \mathrm{~h}$
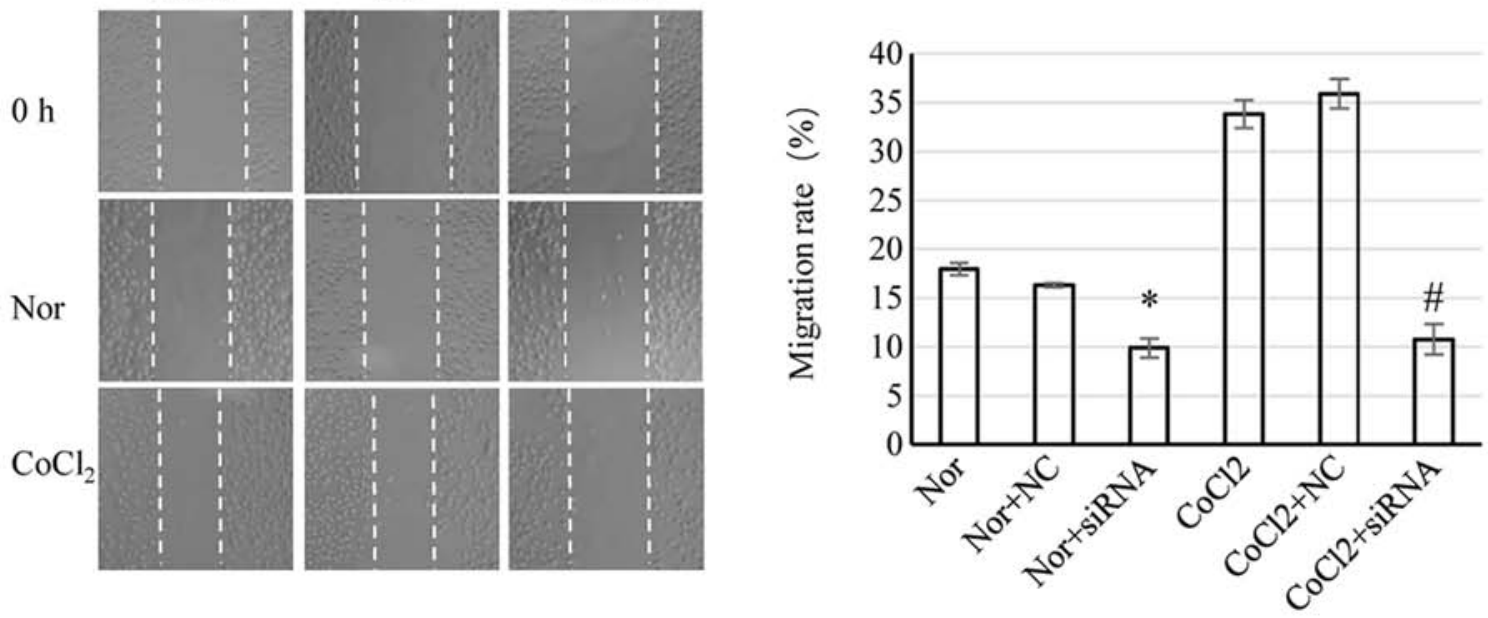

Figure 2. STAT3 regulates EMT induced by hypoxia in ESCC. (A-D) PGCL-GFP-STAT3 siRNA (siRNA) or pGCL-GFP (NC) was transfected into EC-1 cells with Lipofectamine 2000 for $24 \mathrm{~h}$ and incubated in normoxia (nor), or with $\mathrm{CoCl}_{2}(100 \mu \mathrm{mol} / \mathrm{l})$. (A) Relative mRNA expression levels of E-cadherin (E-cad), vimentin (vim), HIF-1 $\alpha$, and STAT3 in ESCC cells, with or without $\mathrm{CoCl}_{2}(100 \mu \mathrm{mol} / \mathrm{l})$ for $24 \mathrm{~h}$. mRNA expression was significantly different from that in the blank control group in normoxia $\left({ }^{*} \mathrm{P}<0.05\right)$. mRNA expression was significantly different from that in the blank control group in hypoxia $\left({ }^{\sharp} \mathrm{P}<0.05\right)$. (B) Western blot analysis of E-cadherin, vimentin, HIF-1 $\alpha$, or pSTAT3 proteins, with or without $\mathrm{CoCl}_{2}(100 \mu \mathrm{mol} / \mathrm{l})$ for $24 \mathrm{~h}$. Results were normalized against $\beta$-actin. (C) IF of E-cadherin, vimentin, and HIF-1 $\alpha$ expression, with or without $\mathrm{CoCl}_{2}(100 \mu \mathrm{mol} / \mathrm{l})$ for $24 \mathrm{~h}$. (D) Cell migration was prevented by STAT3 inhibition. Scratches were made after transfections and the cells were incubated in normoxia, or with $\mathrm{CoCl}_{2}(100 \mu \mathrm{mol} / \mathrm{l})$, for $12 \mathrm{~h}$. The cell migration rate was significantly lower than in the blank control group in normoxia $\left({ }^{*} \mathrm{P}<0.05\right)$. The cell migration rate was significantly lower than in the blank control group in hypoxia $\left({ }^{\#} \mathrm{P}<0.05\right)$.

results indicated that STAT3 knockdown regulates HIF-1 $\alpha$ and obliterates EMT induced by hypoxia. To observe the effect of HIF-1 $\alpha$ in hypoxia-induced EMT, we used HIF-1 $\alpha$ siRNA to block the expression of HIF-1 $\alpha$, and found that, in hypoxia, inhibiting the expression of HIF-1 $\alpha$ increases E-cadherin expression and reduces vimentin expression. Based on the 
$\mathrm{E}$
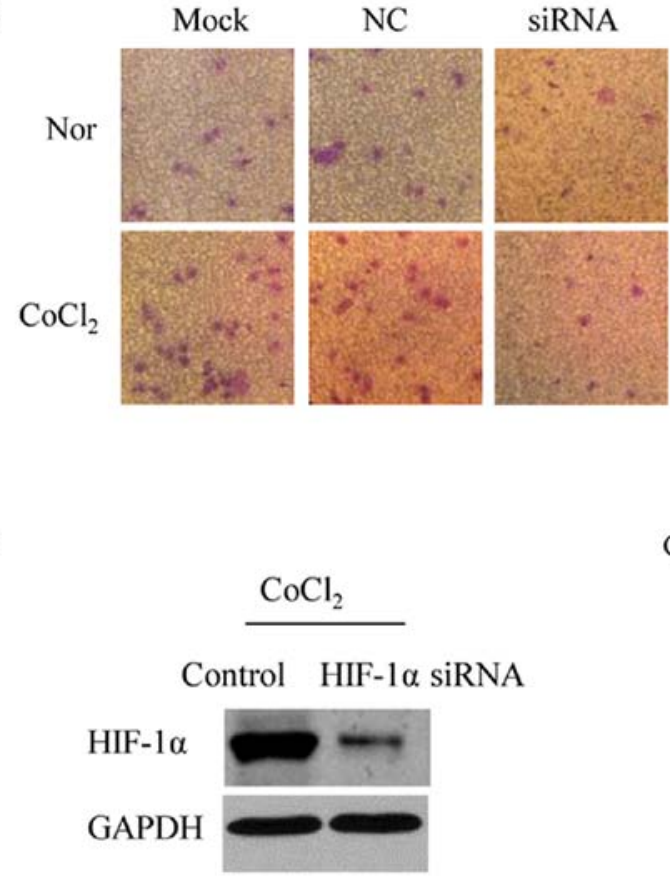

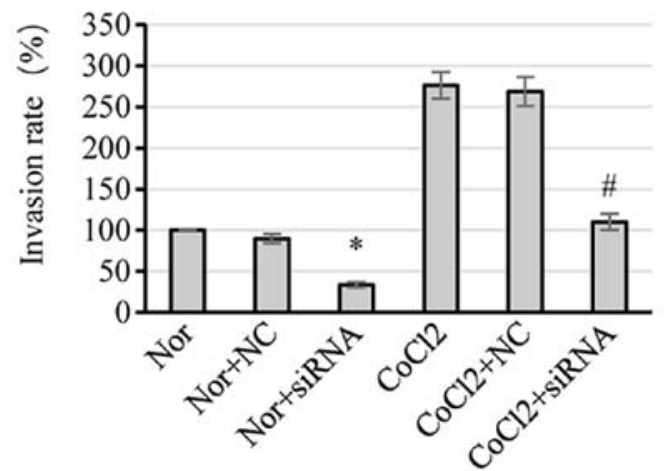

G

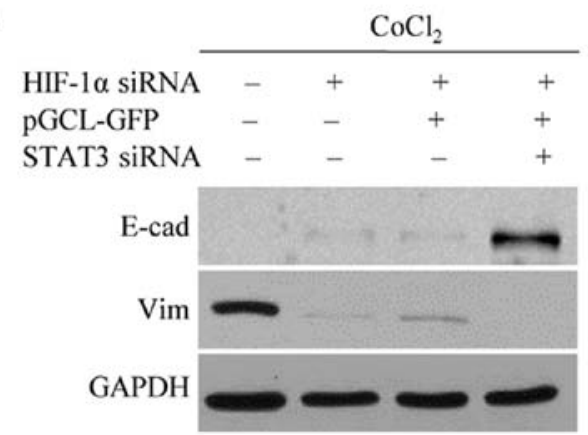

Figure 2 Continued. STAT3 regulates EMT induced by hypoxia in ESCC. (E and G) PGCL-GFP-STAT3 siRNA (siRNA) or pGCL-GFP (NC) was transfected into EC-1 cells with Lipofectamine 2000 for $24 \mathrm{~h}$ and incubated in normoxia (nor), or with $\mathrm{CoCl}_{2}(100 \mu \mathrm{mol} / \mathrm{l})$. (E) Cells invasion was suppressed when STAT3 was inhibited. Transfected cells seeded into invasion chambers and incubated with or without $\mathrm{CoCl}_{2}(100 \mu \mathrm{mol} / \mathrm{l})$ for $12 \mathrm{~h}$, then were stained and detected. The cell invasion rate was significantly lower than in the blank control group in normoxia (" $\mathrm{P}<0.05)$. The cell invasion rate was significantly lower than in the blank control group in hypoxia $\left({ }^{\#} \mathrm{P}<0.05\right)$. The results represent means \pm SD from three independent experiments. (F) HIF-1 $\alpha$ protein expression of EC-1 cells with $\mathrm{CoCl}_{2}(100 \mu \mathrm{mol} / 1$ for $24 \mathrm{~h}$ ) was inhibited by HIF-1 $\alpha$ siRNA, analyzed using western blot method. (G) Western blot analysis of E-cadherin and vimentin protein expression of EC-1 with HIF-1 $\alpha$ siRNA and STAT3 siRNA with $\mathrm{CoCl}_{2}(100 \mu \mathrm{mol} / 1$ for $24 \mathrm{~h})$. GAPDH served as a loading control in (F and G).

A

TE-1

EC-1

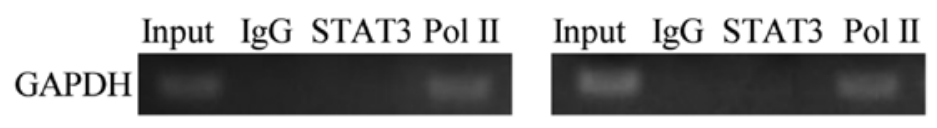

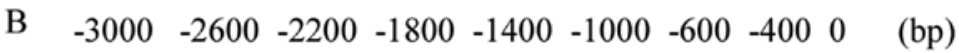

\begin{tabular}{|lllllllll|} 
F1 & F2 & F3 & F4 & F5 & F6 & F7 & F8 & ATG \\
\hline & & & & & & & \\
\hline R1 & R2 & R3 & R4 & R5 & R6 & R7 & R8
\end{tabular}

C

TE-1

EC-1

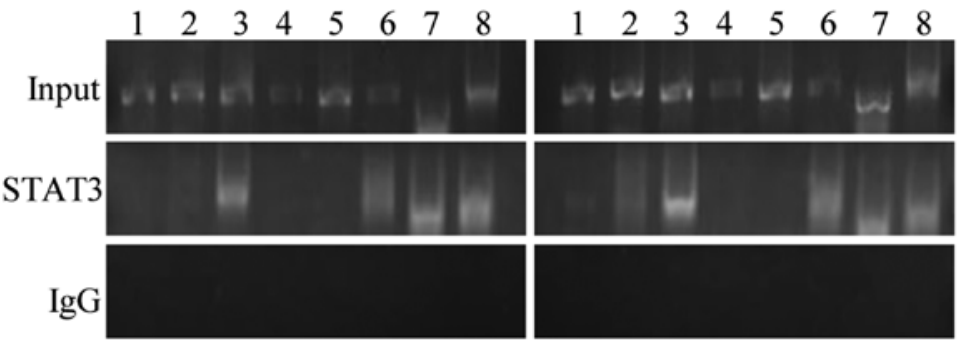

Figure 3. STAT3 binds to the HIF-1 $\alpha$ promoter in ESCC cells. ChIP assays were performed with chromatin extracts from TE-1 and EC-1 cells: (A) RT-PCR to amplifying glyceraldehyde-3-phosphate dehydrogenase (GAPDH) from the input or enriched DNA from immunoprecipitation with IgG (negative control), anti-STAT3, anti-Pol II (positive control) for assay efficacy. (B) The primer locations in the HIF-l $\alpha$ promoter: ATG indicates the beginning of open reading frame (ORF) of HIF-1 $\alpha$ gene. (C) STAT3 binds to the HIF-1 $\alpha$ promoter. The input or immunoprecipitated DNA with IgG or anti-STAT3 antibody was analyzed by PCR to amplify $H I F-1 \alpha$ promoter sequences.

results of blocking HIF-1 $\alpha$, inhibition of STAT3 by STAT3 siRNA can further increase E-cadherin level and reduce vimentin expression (Fig. 2F and G).
STAT3 binds the promoter of HIF-1 $\alpha$ gene. STAT3 is a transcription factor that regulates gene expression and exerts its physiologic functions by binding to DNA. Previous studies 
indicated that STAT3 increases HIF-1 $\alpha$ protein stability and interacts with HIF-1 $\alpha$ to bind to HIF1 target gene promoters. We noted that STAT3 affected mRNA expression of HIF-1 $\alpha$ in ESCC cell lines in normoxia and hypoxia conditions (Fig. 2A). To determine the specific mechanisms of HIF-1 $\alpha$ regulation by STAT3, we used in vivo chromatin immunoprecipitation (ChIP) assays to detect STAT3 binding to the HIF-1 $\alpha$ promoter (Fig. 3). The results in Fig. 3A indicated the efficiency of the immunoprecipitation, and that STAT3 did not bind non-specifically to DNA. Eight pairs of primers were designed, covering $3 \mathrm{kbp}$ upstream of the HIF-1 $\alpha$ open reading frame (Fig. 3B). As shown in Fig. 3C primer pairs 3, 6, 7, and 8 produced visible bands, suggesting that STAT3 binds to regions on the HIF-1 $\alpha$ promoter. The result shows that nuclear STAT3 binds to the HIF-1 $\alpha$ promoter of ESCC cells.

STAT3 siRNA inhibits EMT and tumour progression of ESCC in vivo. STAT3 regulates HIF-1 $\alpha$ expression and hypoxiainduced EMT in ESCC in vitro. For in vivo experiments, athymic nude mice were implanted with xenografted human ESCC tumours. Mice were treated with STAT3 siRNA, or with the controls, empty vector pGCL-GFP or saline. Tumour sizes were smaller in the STAT3 siRNA treated group than in control groups $(\mathrm{P}<0.05)$ (Fig. 4A). Lower expression of p-STAT3, HIF-1 $\alpha$ and vimentin, and higher expression of E-cadherin, were detected in the treatment group than in the control groups. The target proteins did not show differences in the two control groups (Fig. 4B). Taken together, these results indicated that STAT3 influences ESCC tumour proliferation by regulating EMT via HIF-1 $\alpha$ in ESCC in vivo.

\section{Discussion}

Hypoxia is one of the driving factors of EMT, and HIF-1 $\alpha$ is the key factor of this process. Previous studies have demonstrated hypoxia-induced EMT in breast, pancreatic, prostate, and colon cancers. This study demonstrated hypoxia-induced EMT in ESCC for the first time, which occurs through the upregulation of HIF-1 $\alpha$. When HIF-1 $\alpha$ expression is inhibited, hypoxia can no longer induce EMT. In our previous study, we found that STAT3 regulates EMT by targeting the transcription factors TWIST, SLUG, and SNAIL, which regulate E-cadherin expression. Does STAT3 play a role in hypoxiainduced EMT. In this study, we found that EMT in hypoxia is reduced when STAT3 expression is silenced, indicating that STAT3 is important in hypoxia-induced EMT. ESCCs undergo EMT in normoxic conditions, as well, although weaker than in hypoxic condition. The EMT in normoxic condition also was attenuated by inhibiting STAT3, whereas HIF-1 $\alpha$ at a low level. This phenomenon indicated that STAT3 regulates EMT in normoxic and in hypoxic conditions through different pathways. The specific signalling networks involved in STAT3-regulated EMT need further investigation.

HIF- $1 \alpha$ and Stat 3 are overexpressed in diverse cancers and both play key roles in cancer progression. STAT3 affects HIF-1 $\alpha$ expression and works with HIF-1 $\alpha$ to adjust hypoxic reactions. It was found that Stat 3 is required for both basal and growth signal-induced expression of HIF-1 $\alpha$. STAT3 binds to the promoters of HIF1 target genes, interacting with HIF-1 $\alpha$ protein and recruiting a DNA binding complex to promote
A
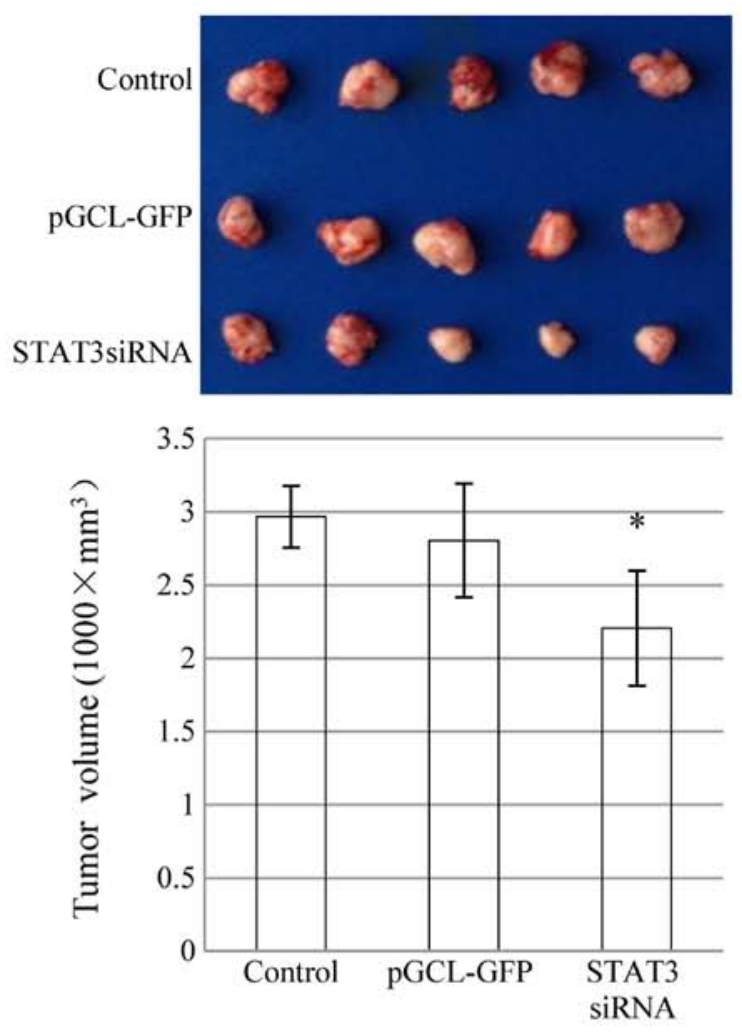

B

$$
\text { Control }
$$

pGCL-GFP

STAT3 siRNA

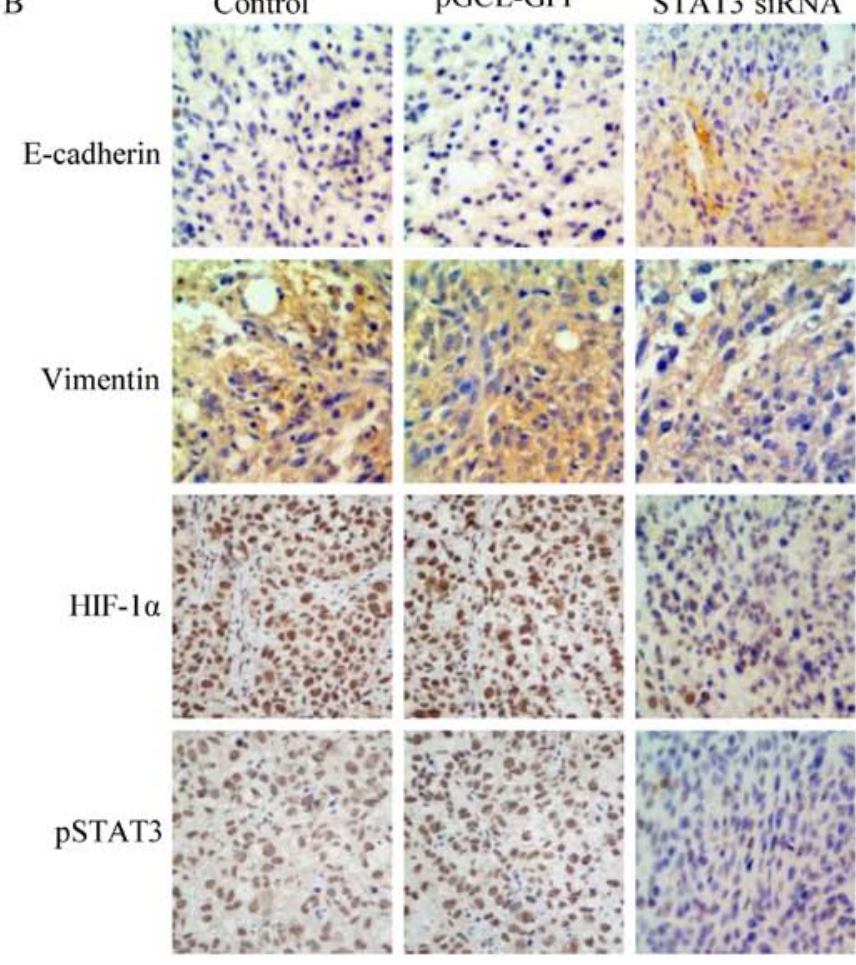

Figure 4. STAT3 siRNA inhibits EMT and tumour progression of ESCC in vivo. ESCC EC-1 cells were implanted subcutaneously into the dorsal regions of mice. Mice were treated with normal saline, empty vector pGCL-GFP (pGCL-GFP), or pGCL-GFP STAT3 siRNA (STAT3 siRNA). (A) Tumours were excised from the mice 4 weeks after implantation. The tumour sizes were significantly smaller than those in blank control group $\left({ }^{*} \mathrm{P}<0.05\right)$. (B) Expression of pSTAT3, HIF-1 $\alpha$, E-cadherin, and vimentin in the xenografts was detected using immunohistochemistry.

transcription. Our study revealed the potential binding of STAT3 to the HIF-1 $\alpha$ promoter in ESCC cells, indicating that 
STAT3 transcriptionally regulates HIF-1 $\alpha$ expression. This is consistent with the results of previous studies (34). The difference is, our study found three potential binding sites in the HIF-1 $\alpha$ promoter, in addition to the Stat3 binding site at position-373, which may play different roles in HIF-1 $\alpha$ transcription. HIF-1 $\alpha$ mRNA levels do not increase in hypoxia, but when STAT3 was silenced, HIF-1 $\alpha$ mRNA declined significantly. Perhaps the role of STAT3 is to maintain normal levels of HIF-1 $\alpha$ mRNA. Does STAT3 interact with HIF-1 $\alpha$ in EMT? Our experiment showed that HIF-1 $\alpha$ and EMT were downregulated by STAT3 siRNA. This suggests that STAT3 affects EMT induced by hypoxia by regulating HIF-1 $\alpha$, which was previously shown to regulate transcriptional regulators of E-cadherin. Taken together with prior studies, STAT3 might adjust EMT by targeting transcriptional regulators of E-cadherin, directly or indirectly.

In conclusion, our study demonstrated, for the first time, a key role of STAT3 in hypoxia-induced EMT in ESCC. Tumour cells acquire stem cell-like and chemoresistant phenotypes from EMT. Our findings may explain the radio/chemo resistance of ESCC and the values of therapy that targets STAT3. Not all tumour cells undergo EMT, and the process rarely occurs during the entire process of tumour progression. When EMT cells arrive at their destination, these mesenchymal cells may undergo the reverse process, mesenchymal-epithelial transition (MET), and then adhere to and proliferate in the new organs. The heterogeneity of malignant tumour cells with mixed epithelial and mesenchymal traits may enhance the ability of these cells to adapt changes in the microenvironments, and to survive and evolve quickly. EMT is not the complete story of tumour progression. Further studies are required to clarify the precise mechanisms and signals that regulate the transition between epithelial and mesenchymal phenotypes, and the involvement of STAT3 and hypoxia in these processes.

\section{References}

1. Książkiewicz M, Markiewicz A and Zaczek AJ: Epithelialmesenchymal transition: A hallmark in metastasis formation linking circulating tumor cells and cancer stem cells. Pathobiology 79: 195-208, 2012.

2. Tania M, Khan MA and Fu J: Epithelial to mesenchymal transition inducing transcription factors and metastatic cancer. Tumour Biol 35: 7335-7342, 2014

3. Imai T, Horiuchi A, Wang C, Oka K, Ohira S, Nikaido T and Konishi I: Hypoxia attenuates the expression of E-cadherin via up-regulation of SNAIL in ovarian carcinoma cells. Am J Pathol 163: 1437-1447, 2003.

4. Philip B, Ito K, Moreno-Sánchez R and Ralph SJ: HIF expression and the role of hypoxic microenvironments within primary tumours as protective sites driving cancer stem cell renewal and metastatic progression. Carcinogenesis 34: 1699-1707, 2013.

5. Yoo YG, Christensen J, Gu J and Huang LE: HIF-1 $\alpha$ mediates tumor hypoxia to confer a perpetual mesenchymal phenotype for malignant progression. Sci Signal 4: pt4, 2011.

6. Sahlgren C, Gustafsson MV, Jin S, Poellinger L and Lendahl U: Notch signaling mediates hypoxia-induced tumor cell migration and invasion. Proc Natl Acad Sci USA 105: 6392-6397, 2008

7. Copple BL: Hypoxia stimulates hepatocyte epithelial to mesenchymal transition by hypoxia-inducible factor and transforming growth factor-beta-dependent mechanisms. Liver Int 30: 669-682, 2010.

8. Li GQ, Zhang Y, Liu D, Qian YY, Zhang H, Guo SY, Sunagawa M, Hisamitsu T and Liu YQ: PI3 kinase/Akt/HIF-1 $\alpha$ pathway is associated with hypoxia-induced epithelial-mesenchymal transition in fibroblast-like synoviocytes of rheumatoid arthritis. Mol Cell Biochem 372: 221-231, 2013.
9. Zhang Q, Bai X, Chen W, Ma T, Hu Q, Liang C, Xie S, Chen C, $\mathrm{Hu} \mathrm{L}, \mathrm{Xu} \mathrm{S}$, et al: Wnt/ $\beta$-catenin signaling enhances hypoxiainduced epithelial-mesenchymal transition in hepatocellular carcinoma via crosstalk with hif-1 $\alpha$ signaling. Carcinogenesis 34: 962-973, 2013.

10. Percio S, Coltella N, Grisanti S, Bernardi R and Pattini L: A HIF-1 network reveals characteristics of epithelial-mesenchymal transition in acute promyelocytic leukemia. Genome Med 6: 84, 2014.

11. Brierley MM and Fish EN: STATs: Multifaceted regulators of transcription. J Interferon Cytokine Res 25: 733-744, 2005.

12. Zhao S, Venkatasubbarao K, Lazor JW, Sperry J, Jin C, Cao L and Freeman JW: Inhibition of STAT3 Tyr705 phosphorylation by Smad4 suppresses transforming growth factor beta-mediated invasion and metastasis in pancreatic cancer cells. Cancer Res 68: 4221-4228, 2008.

13. Colomiere M, Ward AC, Riley C, Trenerry MK, Cameron-Smith D, Findlay J, Ackland L and Ahmed N: Cross talk of signals between EGFR and IL-6R through JAK2/STAT3 mediate epithelialmesenchymal transition in ovarian carcinomas. Br J Cancer 100: 134-144, 2009.

14. Cho KH, Choi MJ, Jeong KJ, Kim JJ, Hwang MH, Shin SC, Park CG and Lee HY: A ROS/STAT3/HIF-1 $\alpha$ signaling cascade mediates EGF-induced TWIST1 expression and prostate cancer cell invasion. Prostate 74: 528-536, 2014.

15. Lo HW, Hsu SC, Xia W, Cao X, Shih JY, Wei Y, Abbruzzese JL, Hortobagyi GN and Hung MC: Epidermal growth factor receptor cooperates with signal transducer and activator of transcription 3 to induce epithelial-mesenchymal transition in cancer cells via up-regulation of TWIST gene expression. Cancer Res 67: 9066-9076, 2007.

16. Huang C, Yang G, Jiang T, Zhu G, Li H and Qiu Z: The effects and mechanisms of blockage of STAT3 signaling pathway on IL-6 inducing EMT in human pancreatic cancer cells in vitro. Neoplasma 58: 396-405, 2011.

17. Xiong H, Hong J, Du W, Lin YW, Ren LL, Wang YC, Su WY, Wang JL, Cui Y, Wang ZH, et al: Roles of STAT3 and ZEB1 proteins in E-cadherin down-regulation and human colorectal cancer epithelial-mesenchymal transition. J Biol Chem 287: 5819-5832, 2012.

18. Hu QD, Chen W, Yan TL, Ma T, Chen CL, Liang C, Zhang Q, Xia XF, Liu H, Zhi X, et al: NSC 74859 enhances doxorubicin cytotoxicity via inhibition of epithelial-mesenchymal transition in hepatocellular carcinoma cells. Cancer Lett 325: 207-213, 2012.

19. Yue P,Zhang X,Paladino D, Sengupta B, Ahmad S, Holloway RW, Ingersoll SB and Turkson J: Hyperactive EGF receptor, Jaks and Stat 3 signaling promote enhanced colony-forming ability, motility and migration of cisplatin-resistant ovarian cancer cells. Oncogene 31: 2309-2322, 2012.

20. Jung JE, Lee HG, Cho IH, Chung DH, Yoon SH, Yang YM, Lee JW, Choi S, Park JW, Ye SK, et al: STAT3 is a potential modulator of HIF-1-mediated VEGF expression in human renal carcinoma cells. FASEB J 19: 1296-1298, 2005.

21. Pawlus MR, Wang L and Hu CJ: STAT3 and HIF1 $\alpha$ cooperatively activate HIF1 target genes in MDA-MB-231 and RCC4 cells. Oncogene 33: 1670-1679, 2014.

22. Xu Q, Briggs J, Park S, Niu G, Kortylewski M,Zhang S, Gritsko T, Turkson J, Kay H, Semenza GL, et al: Targeting Stat3 blocks both HIF-1 and VEGF expression induced by multiple oncogenic growth signaling pathways. Oncogene 24: 5552-5560, 2005.

23. Ardyanto TD, Osaki M, Tokuyasu N, Nagahama Y and Ito H: $\mathrm{CoCl}_{2}$-induced HIF-1alpha expression correlates with proliferation and apoptosis in MKN-1 cells: A possible role for the PI3K/Akt pathway. Int J Oncol 29: 549-555, 2006.

24. Yang G, Huang C, Cao J, Huang KJ, Jiang T and Qiu ZJ: Lentivirus-mediated shRNA interference targeting STAT3 inhibits human pancreatic cancer cell invasion. World J Gastroenterol 15: 3757-3766, 2009.

25. Lakka SS, Rajan M, Gondi C, Yanamandra N, Chandrasekar N, Jasti SL, Adachi Y, Siddique K, Gujrati M, Olivero W, et al: Adenovirus-mediated expression of antisense MMP-9 in glioma cells inhibits tumor growth and invasion. Oncogene 21: 8011-8019, 2002.

26. Santel A, Aleku M, Röder N, Möpert K, Durieux B, Janke O, Keil O, Endruschat J, Dames S, Lange C, et al: Atu027 prevents pulmonary metastasis in experimental and spontaneous mouse metastasis models. Clin Cancer Res 16: 5469-5480, 2010. 
27. Yang HB, Yang X, Cao J, Li S, Liu YN, Suo ZW, Cui HB, Guo Z and $\mathrm{Hu}$ XD: cAMP-dependent protein kinase activated Fyn in spinal dorsal horn to regulate NMDA receptor function during inflammatory pain. J Neurochem 116: 93-104, 2011.

28. Cannito S, Novo E, Compagnone A, Valfrè di Bonzo L, Busletta C, Zamara E, Paternostro C, Povero D, Bandino A, Bozzo F, et al: Redox mechanisms switch on hypoxia-dependent epithelial-mesenchymal transition in cancer cells. Carcinogenesis 29: 2267-2278, 2008.

29. Zhang L, Huang G, Li X, Zhang Y, Jiang Y, Shen J, Liu J, Wang Q, Zhu J, Feng X, et al: Hypoxia induces epithelial-mesenchymal transition via activation of SNAI1 by hypoxia-inducible factor-1 $\alpha$ in hepatocellular carcinoma. BMC Cancer 13: 108, 2013.

30. Matsuyama T, Nakanishi K, Hayashi T, Yoshizumi Y, Aiko S, Sugiura Y, Tanimoto T, Uenoyama M, Ozeki Y and Maehara T: Expression of hypoxia-inducible factor-1alpha in esophageal squamous cell carcinoma. Cancer Sci 96: 176-182, 2005.
31. You Z, Xu D, Ji J, Guo W, Zhu W and He J: JAK/STAT signal pathway activation promotes progression and survival of human oesophageal squamous cell carcinoma. Clin Transl Oncol 14: 143-149, 2012.

32. Yang Y, Pan X, Lei W, Wang J, Shi J, Li F and Song J: Regulation of transforming growth factor-beta 1-induced apoptosis and epithelial-to-mesenchymal transition by protein kinase A and signal transducers and activators of transcription 3 . Cancer Res 66: 8617-8624, 2006.

33. Sullivan NJ, Sasser AK, Axel AE, Vesuna F, Raman V, Ramirez N, Oberyszyn TM and Hall BM: Interleukin-6 induces an epithelial-mesenchymal transition phenotype in human breast cancer cells. Oncogene 28: 2940-2947, 2009.

34. Niu G, Briggs J, Deng J, Ma Y, Lee H, Kortylewski M, Kujawski M, Kay H, Cress WD, Jove R, et al: Signal transducer and activator of transcription 3 is required for hypoxia-inducible factor-1alpha RNA expression in both tumor cells and tumorassociated myeloid cells. Mol Cancer Res 6: 1099-1105, 2008. 